\title{
Nonsaturating magnetoresistance and Hall coefficient reversal in a model composite semiconductor
}

\author{
V. Guttal and D. Stroud \\ Department of Physics, The Ohio State University, Columbus, Ohio 43210-1106, USA \\ (Received 15 November 2005; revised manuscript received 23 December 2005; published 10 February 2006)
}

\begin{abstract}
We calculate the transverse magnetoresistance (TMR) and Hall coefficient $R_{H}$ of a three-dimensional composite medium with both positive and negative charge carriers, using the effective-medium approximation. The composite is assumed to be composed of two types of small crystallites, in which the charge carriers are either all electrons or all holes. The conductivity tensors of the two components are assumed to be of the standard free-electron form. At all nonzero concentrations, the composite is found to have a large, nonsaturating TMR. For a given magnetic field $h$, the TMR is a maximum at the concentration $p^{*}$ where $R_{H}$ changes sign; at this concentration, the TMR may be a factor of hundreds or thousands for realistic magnetic field. We discuss the relevance of these results to recent experiments on silver chalcogenide semiconductors, where similar behavior has been reported as a function of pressure.
\end{abstract}

DOI: 10.1103/PhysRevB.73.085202

PACS number(s): 75.47.De, 61.43.Hv, 72.15.Gd

\section{INTRODUCTION}

Many materials have long been known to exhibit a nonsaturating transverse magnetoresistance (TMR). For example, polycrystalline samples of $\mathrm{Cu}, \mathrm{Ag}$, and $\mathrm{Au}$, and even simple metals such as $\mathrm{K}$, In, and $\mathrm{Al}$, exhibit a TMR that varies linearly with $H$ at high magnetic field $H .{ }^{1}$ In single crystals of materials that have Fermi surfaces with open orbits, the TMR is proportional to $H^{2}$ for certain orientations of $\mathbf{H}$ relative to the crystal axes. ${ }^{2,3}$

Recently, there has been renewed interest in materials showing a linear TMR because they are potentially applicable as magnetic field sensors. In the search for such useful materials, it has been discovered that the doped silver chalcogenides $\mathrm{Ag}_{2+\delta}$ Te and $\mathrm{Ag}_{2+\delta}$ Se exhibits remarkably large and linear TMR over temperatures ranging from 4 to $300 \mathrm{~K}$ and fields as high as $50 \mathrm{~T} .{ }^{4,5}$ Surprisingly, these nonstoichiometric materials are intrinsically nonmagnetic. By contrast, the giant magnetoresistance materials, which also show a huge TMR, generally include ferromagnetic layers or particles. ${ }^{6}$ There is even some indication of nonsaturating longitudinal magnetoresistance in these materials. ${ }^{7}$

Several mechanisms have been suggested for the linear TMR observed in the chalcogenide semiconductors. One proposal is a model of quantum magnetoresistance. ${ }^{8}$ In this model, a linear magnetoresistance would occur in a semiconductor when the band gap approaches zero and the energymomentum dispersion relation becomes linear in momentum. ${ }^{8,9}$ In another model, it is hypothesized that the material is macroscopically inhomogeneous, in the sense that it has a spatially varying conductivity tensor. ${ }^{10-12}$ In one model of this kind, ${ }^{10,11}$ the material is treated as a highly disordered two-dimensional resistor network. A linear TMR is found when the charge carriers have opposite signs and the space-averaged mobility of the system is zero.

Recently, we presented another version of this twodimensional model. ${ }^{12}$ Our model assumes a two-component system, each having a free-electron conductivity tensor but with carriers of opposite signs and mobilities equal in magnitude. When the areal fractions of two components are equal and provided the system has suitably defined symmetry, we showed analytically, using a duality argument, ${ }^{13}$ that the TMR is exactly linear at high $H$. At this same concentration, the Hall coefficient $R_{H}$ is shown to change sign. Both of these features are in good agreement with experiment ${ }^{5}$ and also with numerical simulations. ${ }^{10}$ For systems having an unequal areal fraction of the two components, a calculation using the effective-medium approximation ${ }^{14}$ (EMA) leads to a saturating TMR and a nonzero $R_{H}$. Some other recent work $^{15,16}$ has also suggested, using duality arguments, that there is a connection between a large linear TMR in two dimensions and a change in sign of the high-field Hall coefficient.

The above model applies to a two-dimensional (2D) composite, i.e., a thin film in which the individual grains extend the entire thickness of the film. Indeed, experiments carried out on thin films of $\mathrm{LaSb}_{2}$, a material which may have the required $2 \mathrm{D}$ geometry, do show a nonsaturating and linear TMR (which is, in fact, much larger than that of the chalcogenide semiconductors). ${ }^{17}$ The individual layers in these samples may not, however, be disordered as required for the model of Ref. 12, though the similarities between their measurements and that model are striking. On the other hand the experimental samples of chalcogenides ${ }^{4,5}$ are almost certainly three-dimensional (3D). If they are, in fact, granular, the linear dimensions of the individual grains are probably much smaller than any of the sample dimensions. Hence it would be useful to have similar calculations for the $3 \mathrm{D}$ case.

In this paper, we carry out such calculations for a highly disordered semiconductor in 3D. The semiconductor is modeled as a two-component system. The two components are assumed to be intrinsically nonmagnetic, but to have charge carriers of opposite sign. Since the analog of the exact duality result is not available in $3 \mathrm{D}$, we calculate the TMR and the Hall resistivity $\rho_{e, x y}$ using the EMA. We find that the TMR is nonsaturating at any nonzero concentration, in contrast to the 2D case, but is largest at the critical concentration where a suitably defined average charge carrier density is zero. Just at this point, we find that $\rho_{e, x y}$ changes sign, in qualitative agreement with experiment.

The remainder of this paper is organized as follows. In Sec. II, we describe our model, and the approximation we 
use to treat it. Our numerical results are given in Sec. III. In Sec. IV, we discuss these results, and compare them to the $2 \mathrm{D}$ results, and to experiment.

\section{THE MODEL}

We begin by briefly reviewing the effective medium approximation, which we use to calculate the effective conductivity tensor $\sigma_{e}$ of an inhomogeneous material having a conductivity tensor $\sigma(\mathbf{x})$ which varies randomly with position $\mathbf{x}$. The composite is assumed to be made up of grains of two types $A$ and $B$, which have conductivity tensors $\sigma_{A}$ and $\sigma_{B}$, respectively. To calculate $\sigma_{e}$ within the EMA, one imagines that a uniform electric field $\mathbf{E}_{0}$ is applied to the composite. Then the electric field $\mathbf{E}_{i}$ within the $i$ th inclusion is calculated as if that inclusion were ellipsoidal and embedded in a uniform background conducting material with a conductivity tensor $\sigma_{\mathbf{e}} \cdot \sigma_{e}$ is chosen so that $\langle\mathbf{J}\rangle=\sigma_{\mathbf{e}}\langle\mathbf{E}\rangle$, where the angular brackets denote a spatial average. Physically, $\sigma_{\mathrm{e}}$ is the measured, macroscopic conductivity of the sample. The EMA yields a self-consistent $3 \times 3$ matrix equation for $\sigma_{\mathrm{e}}{ }^{14}$

$$
\left\langle\delta \sigma_{i}\left(I-\Gamma \delta \sigma_{i}\right)^{-1}\right\rangle=0 .
$$

Here $\delta \sigma_{i}=\sigma_{i}-\sigma_{e}$ and $\Gamma$ is a suitable depolarization tensor, which depends on $\sigma_{e}$ and also on the shapes of the grains.

Of course, in a realistic composite medium, the grains will not actually be ellipsoids. The assumption of an ellipsoidal shape is motivated by computational convenience: the depolarization tensor $\Gamma$ is readily calculated if the grains are ellipsoids, ${ }^{14}$ and the EMA is even analytically tractable. In the present work, we make the further assumption that the grains can be approximated as spheres. While the grains will not actually be spherical, we believe that this approximation should be reasonable if the grains are compact and not too anisotropic in shape. We would not expect major qualitative differences in our results if the grains are nonspherical but still ellipsoidal. Finally, the radius of the spheres should have no effect on the transport properties of such a composite, so long as it is small compared to each of the sample dimensions. This condition means that the sample can be described by a suitable effective conductivity tensor.

In the present calculations, we assume that the conductivity tensor for component $A$ is given by

$$
\begin{gathered}
\sigma_{A, x x}=\sigma_{A, y y}=\frac{\sigma_{A, 0}}{1+h^{2}}, \\
\sigma_{A, x y}=-\sigma_{A, y x}=\frac{h \sigma_{A, 0}}{1+h^{2}}, \quad \sigma_{A, z z}=\sigma_{A, 0} .
\end{gathered}
$$

Here $\sigma_{A, 0}$ is the zero-field conductivity and $h=\omega_{A} \tau_{A}$ $=\mu_{A} H / c$ is a dimensionless magnetic field, defined in terms of the cyclotron frequency $\omega_{A}=q_{A} H /\left(m_{A}^{*} c\right)$, the relaxation time $\tau_{A}$, and the mobility $\mu_{A}=q_{A} \tau_{A} / m_{A}^{*}$ of the type- $A$ charge carrier, which has charge $q_{A}$ and effective mass $m_{A}^{*}$. All other components of $\sigma_{A}$ are zero. For definiteness, we take $q_{A}>0$. Similarly, for the second component, we take the nonzero components to be

$$
\begin{gathered}
\sigma_{B, x x}=\sigma_{B, y y}=\frac{\sigma_{B, 0}(k)}{1+k^{2} h^{2}}, \\
\sigma_{B, x y}=-\sigma_{B, y x}=\frac{k h \sigma_{B, 0}(k)}{1+k^{2} h^{2}}, \quad \sigma_{B, z z}=\sigma_{B, 0} .
\end{gathered}
$$

Thus, $\sigma_{B}$, like $\sigma_{A}$, describes a free-carrier conductor, but with a mobility $\mu_{B}=k \mu_{A}$. Our sign convention is such that if $k$ $=-2$, for example, component $B$ has a charge carrier opposite in sign to that of $A$, but with twice the mobility of the $A$ carriers. In general, the zero-field conductivity $\sigma_{B, 0}(k)$ can depend on $k$. In our calculations below, we consider two models consistent with Eqs. (2)-(5).

Model $I$. In this case, we assume that $\sigma_{B, 0} / \sigma_{A, 0}=|k|$. The physical meaning of this assumption is that the two components have equal charge carrier density. Hence, the ratio of their zero-field conductivities equals the ratio of their mobilities.

Model II. This model is designed to find out the effect of having different charge densities in components $A$ and $B$. To do this, we have assumed $n_{A}=|k| n_{B}$, and also that $\sigma_{A, 0}$ $=\sigma_{B, 0}$. These two assumptions imply that $\mu_{B}=k \mu_{A}$, as in model I.

In a real composite semiconductor with both positive and negative charge carriers, it seems likely that both the carrier densities and the mobilities can be varied independently in the two constituents. Thus, neither model I nor model II is perfectly realistic. However, we believe that model II is more plausible for a real material than model I, because it allows for components with different carrier densities. We have chosen to present results for these two models because they isolate specific features of interest. Note also that for both the above models, both the constituents of our disordered material are nonmagnetic - that is, they have zero TMR in pure form.

We now calculate the effective conductivity tensor for this system for various special cases. We assume the following form for the effective conductivity tensor:

$$
\sigma_{e}=\left(\begin{array}{ccc}
\sigma_{e, x x} & \sigma_{e, x y} & 0 \\
-\sigma_{e, x y} & \sigma_{e, x x} & 0 \\
0 & 0 & \sigma_{e, z z}
\end{array}\right) .
$$

If components $A$ have volume fractions $p_{A}$ and $p_{B}(=1$ $\left.-p_{A}\right)$, respectively, then the self-consistency condition reduces to three coupled algebraic equations for the three independent components $\sigma_{e, x x}, \sigma_{e, x y}$, and $\sigma_{e, z z}$; the remaining nonzero components are $\sigma_{e, y y}=\sigma_{e, x x}$ and $\sigma_{e, y x}=-\sigma_{e, x y}$. For the present model, the coupled self-consistent equations can be written in matrix form as

$$
\sum_{i=A, B} p_{i} \delta \sigma_{i}\left(I-\Gamma \delta \sigma_{i}\right)^{-1}=0
$$

Here $\Gamma$ is the three-dimensional depolarization tensor, as described in Ref. 14. For the present calculations, as mentioned earlier we approximate the grains as spherical, in which case $\Gamma$ can readily be found explicitly. ${ }^{14}$ The coupled equations can then be solved numerically. We have carried out this solution using MATHEMATICA. 


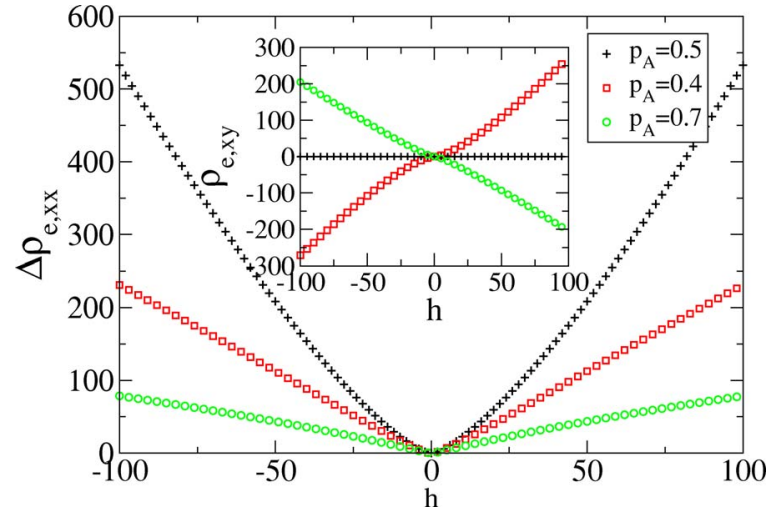

FIG. 1. (Color online) EMA results for a 3D inhomogeneous conductor having two components with equal zero-field conductivities $\sigma_{A, 0}$ and $\sigma_{B, 0}$ and mobilities of equal magnitude $\left|\mu_{A}\right|=\left|\mu_{B}\right|$, but charge carriers of opposite sign, calculated for several values of the volume fraction $p_{A}$. Main part of figure: transverse magnetoresistance $\Delta \rho_{e, x x}\left(p_{A}, h\right) \equiv\left[\rho_{e, x x}\left(p_{A}, h\right)-\rho_{e}\left(p_{A}, 0\right)\right] / \rho_{e}\left(p_{A}, 0\right)$. Inset: Hall resistivity $\rho_{e, x y}\left(p_{A}, h\right)$ [plotted in units of $\left.\rho_{e}\left(p_{A}, 0\right)\right]$.

Given the solutions of Eq. (7), we extract two quantities relevant to experiment. The first is the TMR $\Delta \rho_{e, x x}(h)$, defined by

$$
\Delta \rho_{e x x}(h)=\frac{\rho_{e, x x}(h)-\rho_{e, x x}(0)}{\rho_{e, x x}(0)} .
$$

Here $\rho_{e, i j}(h)$ is the $i j$ th component of the effective resistivity tensor $\rho_{e}(h) \equiv\left[\sigma_{e}(h)\right]^{-1}$. The other quantity of interest is a scaled Hall coefficient $R_{H, e}(h)$, defined by

$$
R_{H, e}(h)=\frac{\rho_{e, x y}(h)}{h},
$$

where $\rho_{e x y}$ is the Hall resistivity. The longitudinal magnetoresistance $\Delta \rho_{e, z z}(h)=\left[\rho_{e z z}(h)-\rho_{e z z}(0)\right] / \rho_{e, z z}(0)$ is zero if $\sigma_{A, 0}=\sigma_{B, 0}$, and is small and positive, but saturating, if $\sigma_{A, 0}$ $\neq \sigma_{B, 0}$.

\section{RESULTS}

We have carried out a series of calculations for binary composites described by model I and by model II. The purpose of these calculations is to obtain the TMR and the Hall resistivity for each composite, as a function of volume fraction $p_{A}$ and magnetic field $h$. We can then qualitatively compare these calculated results with previous results in two dimensions, and with experiment.

We first assume that $\sigma_{A}$ and $\sigma_{B}$ are given by Eqs. (2)-(5), and by model I, i.e., that $\sigma_{B, 0} / \sigma_{A, 0}=|k|$. In Fig. 1, we show the calculated TMR $\Delta \rho_{e, x x}\left(p_{A}, h\right)$ and Hall resistivity $\rho_{e, x y}\left(p_{A}, h\right)$ for several concentrations, all using model I with $k=-1$. With these choices, the two components have equal zero-field conductivities and equal mobilities, and charge carriers of equal and opposite charge. The main part of the figure shows the TMR versus dimensionless field $h$ for several volume fractions $p_{A}$ of component $A$; the inset shows the Hall resistivity $\rho_{e, x y}\left(p_{A}, h\right)$ versus $h$ for several values of $p_{A}$.

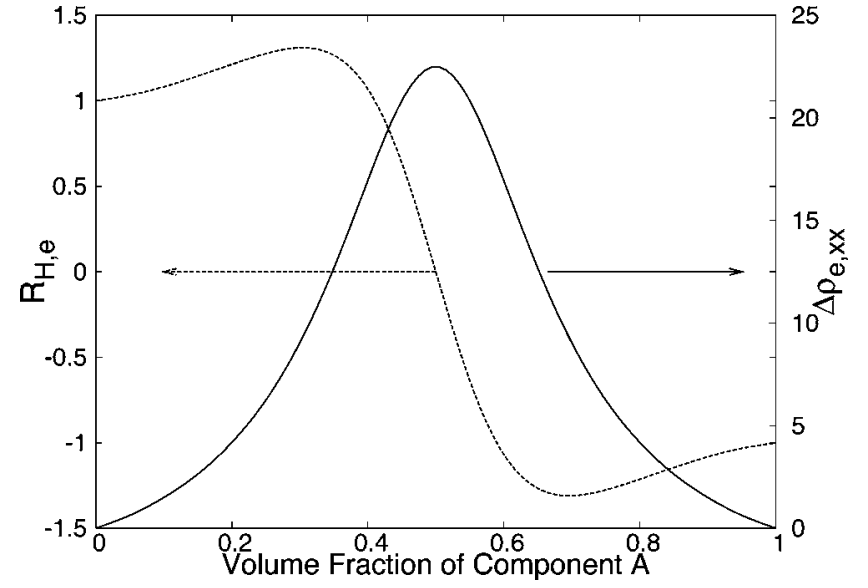

FIG. 2. TMR $\Delta \rho_{e, x x}\left(p_{A}, h\right)$ (right-hand scale) and Hall coefficient $R_{H, e}\left(p_{A}, H\right)$ as defined in Eq. (9) (left-hand scale) plotted as functions of $p_{A}$ for model I, with $k=-1$ and $h=10$. Model I corresponds to $\sigma_{B, 0} / \sigma_{A, 0}=|k|$.

In contrast to the $2 \mathrm{D}$ case, ${ }^{12}$ we see that $\Delta \rho_{e, x x}$ for this model is nonsaturating for all concentrations except for $p_{A}$ $=0$ and 1 , though it is largest at $p_{A}=0.5$ for any value of $h$. For this concentration we find numerically that $\Delta \rho_{e, x x} \approx h^{4 / 3}$. For other concentrations, the TMR for any given $h$ is smaller than that at $p_{A}=0.5$ and appears not to follow a simple power law. At no concentration do we find a $\Delta \rho_{e, x x}$ which is exactly linear in $h$, though $\Delta \rho_{e, x x}$ does appear linear over a broad field range. This behavior is in contrast to that found in $2 \mathrm{D}$, where $\Delta \rho_{e, x x}$ is exactly linear in $h$ for $p_{A}=0.5$, and saturates for other values of $p_{A}$. From Fig. 1 we also see that the Hall resistivity is zero for $p_{A}=1 / 2$, while the Hall coefficient $R_{H, e}=\rho_{e, x y}(h) / h$ is positive for $p_{A}=0.4$ and negative for $p_{A}$ $=0.7$. This suggests that $R_{H, e}$ changes sign at $p_{A}=0.5$, as is confirmed in the next figure.

In Fig. 2, we show $\Delta \rho_{e, x x}\left(p_{A}, h\right)$ and the Hall coefficient $R_{H, e}\left(p_{A}, h\right)$ [Eq. (9)] as functions of $p_{A}$ for fixed $h=10$, using the same model as Fig. 1 . The figure clearly shows that $R_{H, e}$ changes sign at the same value of $p_{A}$ where $\Delta \rho_{e, x x}$ peaks. As the field increases further, the rapid change in $R_{H}$ occurs over a narrower and narrower concentration range. All these features are similar to those obtained previously in 2D.

We have calculated $\Delta \rho_{e, x x}\left(p_{A}, h\right)$ and $R_{H, e}\left(p_{A}, h\right)$ for other values of $k$ within model I. For any fixed $h$, and for any negative value of $k$, we find that $\Delta \rho_{e, x x}$ has a maximum at $p_{A}=1 / 2$ and $R_{H, e}$ changes sign at $p_{A}=1 / 2$. (For $k>0$ the two charge carriers have the same sign and $R_{H, e}$ never passes through zero.) This behavior stands in contrast to model II (discussed below), where the maximum and crossover occur at volume fractions different from $1 / 2$. For model I, the critical concentration can be understood straightforwardly: when $k<0$, the $A$ and $B$ components have charge carriers of opposite sign but the same charge density. Hence, the critical concentration is just that where the space-averaged charge density vanishes. This is also the case for model II, as discussed below.

Next, we consider results obtained from model II. As an example, the predictions of model II at $p_{A}=0.7$ are shown in Fig. 3 for different values of the mobility ratio $k$. In the main 


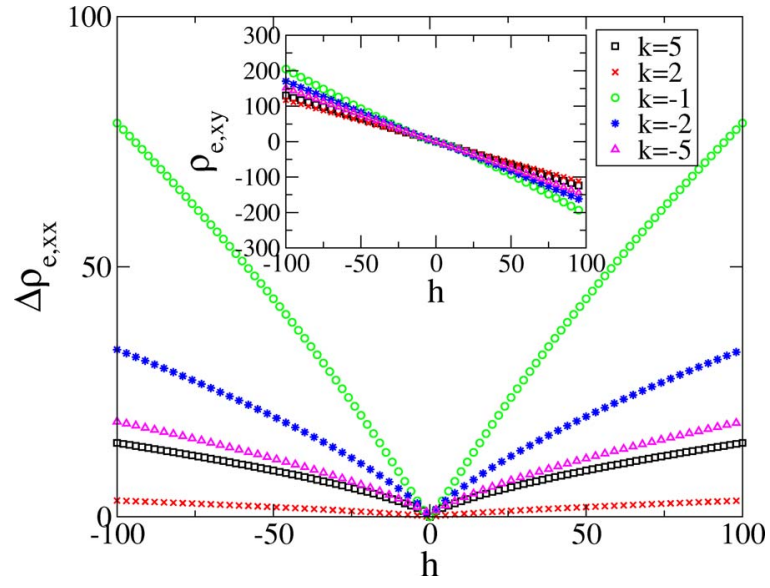

FIG. 3. (Color online) TMR $\Delta \rho_{e . x x}\left(p_{A}, h\right)$ (main part of figure) and $\rho_{e, x y}\left(p_{A}, h\right)$ (inset) for different values of $k$ in model II, taking $\sigma_{A, 0}=\sigma_{B, 0}$ and $p_{A}=0.7$. In model II, $\sigma_{A, 0}$ and $\sigma_{B, 0}$ are independent of $k$.

part of the figure, we show the TMR $\Delta \rho_{e, x x}\left(p_{A}, h\right)$ for this model and concentration, as a function of $h$. Once again, as in model I, the TMR is very large at high $|h|$, but not strictly linear. The Hall resistivities $\rho_{e, x y}\left(p_{A}, h\right)$ for different values $k$ (shown in the inset) are those expected for the majority charge carriers. They depend slightly, but not strongly, on the mobility ratio, given by $k$.

To make the difference between models I and II clearer, we consider as an example the case $k=-5$ in more detail. Figure 4 shows the Hall resistivity $\rho_{e, x y}\left(p_{A}, h\right)$ for various $p_{A}$ near $p_{A}=1 / 6$. Clearly, although the low-field behavior of $\rho_{e, x y}\left(p_{A}, h\right)$ is complicated, the asymptotic high-field behavior of $\rho_{e, x y}\left(p_{A}, h\right)$ is simpler: $\rho_{e, x y}\left(p_{A}, h\right)$ changes sign near $p_{A}=1 / 6$. This asymptotic high-field behavior has a fairly simple interpretation which is discussed below.

In Figs. 5 and 6, we show $\Delta \rho_{e, x x}$ and the Hall coefficient $R_{H, e}$ as functions of $p_{A}$ for the same case $(k=-5), h=100$ and 1000. In both cases, we take $\sigma_{A, 0}=\sigma_{B, 0}$. Quite strikingly in both cases, $\Delta \rho_{e, x x}$ peaks exactly at the value of $p_{A}$ where the high-field $R_{H}$ changes sign, in qualitative agreement with the

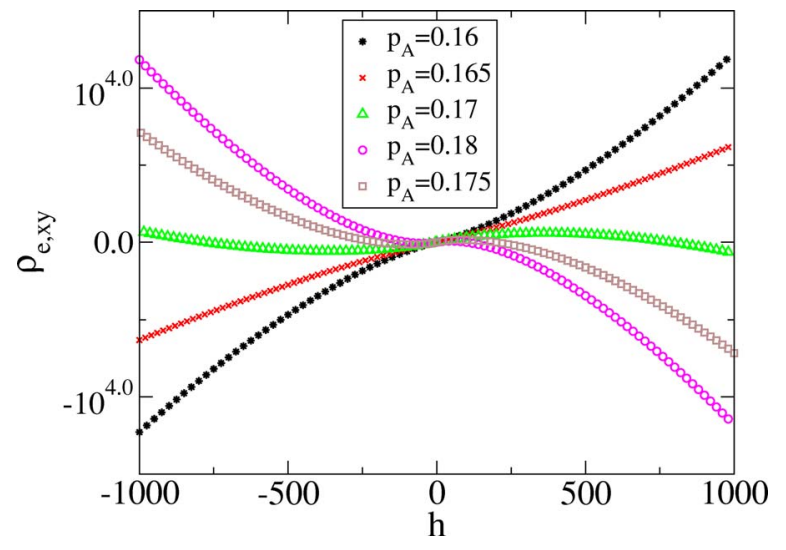

FIG. 4. (Color online) $\rho_{e, x y}\left(p_{A}, h\right)$ plotted as a function of $h$ for several values of $p_{A}$ in model II, all with $k=-5$ and $\sigma_{A, 0}=\sigma_{B, 0}=1$. For this value of $k$, the high-field value of $\rho_{e, x y}$ changes sign at $p_{A}=1 / 6$.

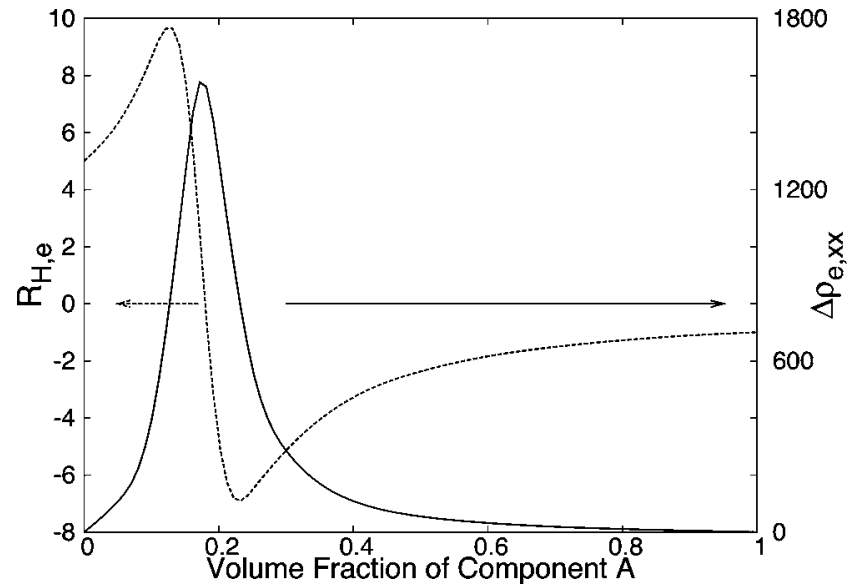

FIG. 5. TMR $\Delta \rho_{e, x x}\left(p_{A}, h\right)$ (right-hand scale) and $R_{H, e}\left(p_{A}, h\right)$ (left-hand scale) as a function of $p_{A}$ for model II, with $h=100, k$ $=-5$, and $\sigma_{A, 0}=\sigma_{B, 0}=1$.

experiments of Lee et al. ${ }^{5}$ Figures 5 and 6 are similar in that $\Delta \rho_{e, x x}\left(p_{A}, h\right)$ has a sharp maximum at $p_{A}=p_{A}^{*}$, where $R_{H, e}\left(p_{A}, h\right)$ changes sign, but the peak is much sharper in Fig. 6 than in Fig. 5. Unlike in model I, $p_{A}^{*}$ depends on $k$.

The critical value $p_{A}^{*}$ can readily be understood as follows. From our numerical results, in Figs. 4-6 and also in other calculations that we do not display here, $p_{A}^{*}=1 /(|k|+1)$ when $k<0$, and there is no such threshold when $k>0$. Now, in model II, the charge carrier density in component $B$ is simply $k$ times its value in component $A$. Thus, if the carriers have opposite signs, the space-averaged charge density vanishes at $p_{A}=p_{A}^{*}$, just the concentration where $R_{H}$ changes sign.

Another striking feature visible in Figs. 5 and 6 is that, for the finite values of $h$ shown, there seems to be some tendency toward a divergence on either side of $p_{A}^{*}$, but the actual calculated $R_{H}$, for any finite $h$, goes through zero at $p_{A}=p_{A}^{*}$. Corresponding to this behavior of $R_{H}$, as already mentioned, there is a peak at $p_{A}^{*}$ in $\Delta \rho_{e, x x}\left(p_{A}, h\right)$ as a function of $p_{A}$ for fixed $h$; the half-width of this peak becomes narrower as $h$ increases.

The results we find from both model I and model II can be briefly summarized as follows. First, $\Delta \rho_{e, x x}\left(p_{A}, h\right)$ is a non-

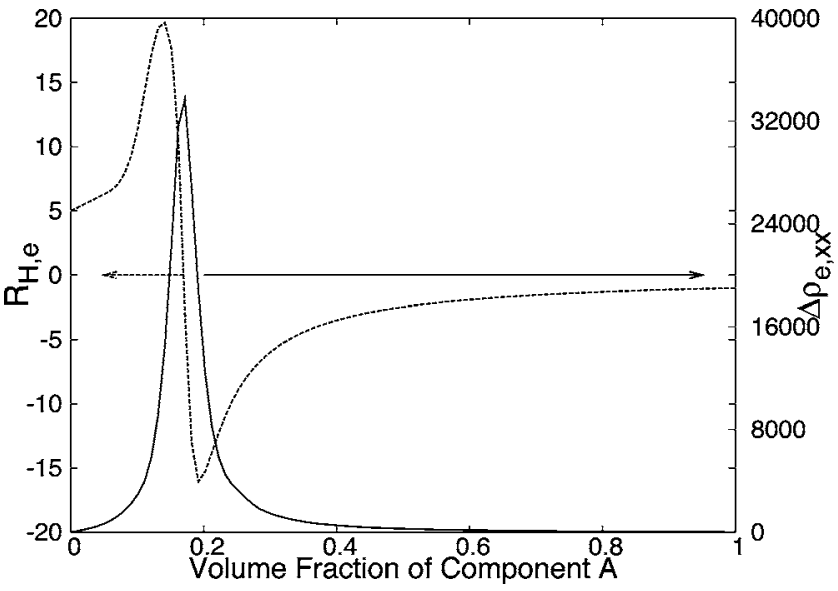

FIG. 6. Same as Fig. 5, except that $h=1000$. 
saturating function of $h$ for any value of $p_{A}$ other than 0 and 1 , but is maximum at the value of $p_{A}$ where $R_{H, e}$ changes sign. Second, the high-field $R_{H, e}$ changes sign just at the concentration $p_{A}^{*}$ where the space-averaged charge density vanishes.

\section{DISCUSSION AND CONCLUSIONS}

Some of the results obtained from both model I and model II are in rough agreement with the experiments of Lee $\mathrm{et} \mathrm{al.}{ }^{5}$ We do not find a precisely linear TMR at any concentration $p_{A}$, though for some concentrations the calculated TMR does appear quite linear over a broad field range. By contrast, Lee et al. report a linear TMR over a broad field range at a pressure where $R_{H}$ changes sign. Also, consistent with Lee et al., we find a $R_{H}$ which changes sign at the concentration where $\Delta \rho_{x x}(h)$ is maximum. But the sharp peaks in $R_{H}$ that we see on either side of $p_{A}^{*}$, particularly at high fields, are not evident in the experiments, though the experiments do show some slight peaks of this kind near the pressure at which $R_{H}$ changes sign.

In our model, the nonsaturating nature of the TMR for all concentrations is due to the current distortions resulting from the inhomogeneity of the two-component system. If spherical inclusions (conducting or nonconducting) are introduced into a three-dimensional conducting medium, the current is strongly distorted around the inclusion. ${ }^{18}$ The TMR produced by such an isolated inclusion is known to be strictly linear for small impurity concentrations. ${ }^{18,19}$ For an inclusion with typical linear dimension $R_{0}$, these current distortions extend a distance of order $h R_{0}$ for high magnetic fields $h$ in the direction parallel to $h$. Since these current shadows extend quite a large distance, even a few inclusions can produce a substantial TMR. ${ }^{20-22}$ For the large concentrations of inclusions considered here, interactions between these current distortions become important even at moderate fields, and hence the TMR produced is typically huge, but not perfectly linear in $h$. Similar not perfectly linear results have been obtained previously using other calculations within the EMA, ${ }^{23}$ but for models in which all carriers have the same sign; in these cases, of course, $R_{H}$ did not change sign at a concentration of maximum TMR.

A striking feature of our results is the sharp peaks in $R_{H . e}$ for model II, on either side of the critical concentration $p_{c}^{*}$ where $R_{H, e}$ passes through zero (see Figs. 6 and 7). The origin of these peaks is not clear, but we may speculate that they come about as follows. In a simple homogeneous conductor at high fields, $R_{H}$ approaches the scatteringindependent value $1 /(n|e| c)$, where $n$ is the net density of charge carriers (density of holes minus that of electrons). In our model II, $n$ passes through zero at $p_{c}^{*}$. Thus, two peaks would be expected as $n$ becomes small in magnitude near $p_{c}^{*}$. The reason that the peaks do not actually diverge is presumably related to the two-component nature of our composite. These two peaks do not appear to be present in the experiments, though there is some indication of slight increases in $\left|R_{H}\right|$ on either side of the critical pressure. ${ }^{5}$ Possibly they would become more apparent at higher fields than those probed in the experiments.

The fact that the current distortions propagate in the longitudinal direction, and have almost no effect in the transverse directions, also explains why we observe saturating TMR in the 2D case with field perpendicular to the film, except for $p_{A}=1 / 2$. For an effectively $2 \mathrm{D}$ conductor of this kind, current distortions cannot propagate, and the TMR should generally saturate. The only exception appears to be when there is some diverging length scale in the disordered system, as happens at the percolation threshold $p_{c}=1 / 2$ in the two-dimensional case.

In the case of model II, our calculations suggest that the TMR will be maximized, and the high-field $R_{H}$ will pass through zero, at a volume fraction of inclusions such that the average carrier density is zero. For model II, this condition is, in fact, equivalent to having the average inverse mobility $\langle 1 / \mu\rangle=p_{A} / \mu_{A}+\left(1-p_{A}\right) / \mu_{B}=0$, where $\mu_{B}=-|k| \mu_{A}$. These observations might be used as guiding principles to develop new materials with a very large TMR.

In summary, we have calculated the effective TMR and Hall coefficient of a macroscopically inhomogeneous medium, using the effective-medium approximation. We find that, when a suitably defined average inverse mobility is zero, the TMR at fixed $h$ has a peak and the corresponding $R_{H}$ has a change of sign. Our results may be relevant to recent experiments on heavily doped silver chalcogenide semiconductors, ${ }^{4,5}$ though our numerical results differ in detail from the measured transport coefficients.

\section{ACKNOWLEDGMENTS}

This work was supported by NSF Grant No. DMR0413395, and benefited from the computational facilities of the Ohio Supercomputer Center.
${ }^{1}$ J. Babiskin and P. G. Siebenmann, Phys. Rev. Lett. 27, 1361 (1971); J. C. Garland and R. Bowers, Phys. Rev. 188, 1121 (1969); W. Kesternich and H. Ullmaier, Phys. Lett. 36A, 411 (1971).

${ }^{2}$ For a discussion, see, e.g., N. W. Ashcroft and N. D. Mermin, Solid State Physics (Saunders, Fort Worth, 1975), Chap. 12.

${ }^{3}$ See, e. g., J. M. Ziman, Principles of the Theory of Solids (Cambridge University Press, Cambridge, U.K., 1972), pp. 250-254.
${ }^{4}$ R. Xu, A. Husmann, T. F. Rosenbaum, M. L. Saboungi, J. E. Enderby, and P. B. Littlewood, Nature (London) 390, 57 (1997).

${ }^{5}$ M. Lee, T. F. Rosenbaum, M.-L. Saboungi, and H. S. Schnyders, Phys. Rev. Lett. 88, 066602 (2002).

${ }^{6}$ See, for example, M. N. Baibich, J. M. Broto, A. Fert, F. Nguyen Van Dau, F. Petroff, P. Etienne, G. Creuzet, A. Friederich, and J. Chazelas, Phys. Rev. Lett. 61, 2472 (1988).

${ }^{7}$ J. Hu, T. F. Rosenbaum, and J. B. Betts, Phys. Rev. Lett. 95, 
186603 (2005).

${ }^{8}$ For a recent short review, see, e.g., A. A. Abrikosov, Europhys. Lett. 49, 789 (2000), and references therein.

${ }^{9}$ A. A. Abrikosov, Phys. Rev. B 58, 2788 (1998).

${ }^{10}$ M. M. Parish and P. B. Littlewood, Nature (London) 426, 162 (2003).

${ }^{11}$ M. M. Parish and P. B. Littlewood, Phys. Rev. B 72, 094417 (2005).

${ }^{12}$ V. Guttal and D. Stroud, Phys. Rev. B 71, 201304 (R) (2005).

${ }^{13}$ K. S. Mendelson, J. Appl. Phys. 46, 4740 (1975).

${ }^{14}$ D. Stroud, Phys. Rev. B 12, 3368 (1975).

${ }^{15}$ S. A. Bulgadaev and F. Kusmartsev, Phys. Lett. A 342, 188 (2005).

${ }^{16}$ S. A. Bulgadaev, Phys. Lett. A 344, 280 (2005).

${ }^{17}$ D. P. Young, R. G. Goodrich, J. F. DiTusa, S. Guo, P. W. Adams,
Julia Y. Chan, and Donavan Hall, Appl. Phys. Lett. 82, 3713 (2003).

${ }^{18}$ J. B. Sampsell and J. C. Garland, Phys. Rev. B 13, 583 (1976).

${ }^{19}$ D. Stroud and F. P. Pan, Phys. Rev. B 13, 1434 (1976).

${ }^{20}$ Tineke Thio and S. A. Solin, Appl. Phys. Lett. 72, 3497 (1998).

${ }^{21}$ S. A. Solin, T. Thio, D. R. Hines, and J. J. Heremans, Science 289, 1530 (2000).

${ }^{22}$ J. Moussa, L. R. Ram-Mohan, J. Sullivan, T. Zhou, D. R. Hines, and S. A. Solin, Phys. Rev. B 64, 184410 (2001).

${ }^{23}$ See, for example, A. K. Sarychev, D. J. Bergman, and Y. M. Strelniker, Phys. Rev. B 48, 3145 (1993); D. J. Bergman and D. G. Stroud, ibid. 62, 6603 (2000); Y. M. Strelniker and D. J. Bergman, ibid. 67, 184416 (2003); D. Stroud and F. P. Pan, ibid. 20, 455 (1979). 